\title{
Transatlantica
}

Revue d'études américaines. American Studies Journal

Line Breaks in America: the Odds and Ends of Poetry

\section{« De quoi ça parle »}

Hommage à Louise Glück, Prix Nobel de littérature 2020

\section{Marie Olivier}

\section{(2) OpenEdition}

\section{Journals}

Édition électronique

URL : https://journals.openedition.org/transatlantica/16688

DOI : $10.4000 /$ transatlantica. 16688

ISSN : 1765-2766

Éditeur

Association française d'Etudes Américaines (AFEA)

Référence électronique

Marie Olivier, « « De quoi ça parle » », Transatlantica [En ligne], 1 | 2021, mis en ligne le 01 juin 2021, consulté le 02 février 2023. URL : http://journals.openedition.org/transatlantica/16688 ; DOI : https:// doi.org/10.4000/transatlantica. 16688

Ce document a été généré automatiquement le 2 février 2023

\section{cc) (†) $\ominus$}

Creative Commons - Attribution - Pas d'Utilisation Commerciale - Pas de Modification 4.0 International - CC BY-NC-ND 4.0

https://creativecommons.org/licenses/by-nc-nd/4.0/ 


\section{«De quoi ça parle »}

Hommage à Louise Glück, Prix Nobel de littérature 2020

\section{Marie Olivier}

1 Lors des différents entretiens journalistiques qui m'ont été demandés suite au Prix Nobel décerné à Louise Glück le 8 octobre 2020, revenait inlassablement la même question: "De quoi parle sa poésie?». La simplicité de la question est à la fois redoutable et désarmante car elle impose nécessairement de thématiser l'œuvre plutôt que d'en explorer véritablement les caractéristiques esthétiques et littéraires. « De quoi ça parle ». Depuis Sartre, puis Barthes après lui, la transitivité de l'écriture qu'une telle question présuppose n'est pas sans être problématique, notamment pour un auteur contemporain, poète par ailleurs, et d'autant plus lorsque celle-ci se nomme Louise Glück. Plutôt que de littéralement évoquer "de quoi ça parle", je souhaiterais rendre hommage à celle dont la voix, selon l'académie suédoise, est si « caractéristique [et] qui, avec une beauté austère, rend l'existence individuelle universelle", en commençant par questionner d'où parlent les voix plurielles de Glück, d'où elles partent pour parler.

2 À quelques exceptions près, les poèmes de Louise Glück mettent en scène la présence d'un sujet au monde qui se veut universel, ni féminin, ni masculin - son écriture fondant la définition de soi davantage dans la négation que l'affirmation. Ses poèmes partent toujours de l'expérience la plus intime - celle de la souffrance, du deuil, de la beauté d'instants qui soudain, scintillent, dans la fugacité d'un mot, d'une image, pour parvenir à atteindre une dimension universelle, comme Stockholm a su le formuler.

3 Dans le discours écrit à l'occasion du prix Nobel, la poète elle-même convoque une autre voix pour parler, ou ne pas parler d'elle, et ainsi, déclare à propos d'Emily Dickinson : "I am talking about a temperament that distrusts public life or sees it as the realm in which generalization obliterates precision, and partial truth replaces candor and charged disclosure" (Glück 2020). Le prix à payer pour le Prix Nobel semble onéreux pour une poète qui a toujours souhaité privilégier la relation intime au lecteur, n'affectionne pas particulièrement les lectures en public, et n'accorde que de très rares entretiens. Depuis l'enfance, Glück a toujours affirmé être attirée par les poèmes dont la voix est solitaire, et abhorrer la grandiloquence de poètes qui s'écouteraient parler (Glück 2020) : 
I was drawn, then as now, to the solitary human voice, raised in lament or longing. And the poets I returned to as I grew older were the poets in whose work I played, as the elected listener, a crucial role. Intimate, seductive, often furtive or clandestine. Not stadium poets. Not poets talking to themselves.

Dans le même texte ${ }^{1}$, Glück confie sa prédilection pour une poésie qui exige du lecteur une présence, une participation active. Formulé ainsi, on serait tenté de croire qu'il s'agirait en d'autres termes de faire 'simplement' l'apologie du texte scriptible. Écrite dans la langue vernaculaire américaine, et d'une simplicité confondante, l'écriture glückienne appelle néanmoins l'une de ces lectures que Barthes a qualifiée d'«aristocratique » qui requiert du lecteur de «ne pas dévorer, ne pas avaler, mais brouter, tondre avec minutie » (Barthes 225). Or, le paradigme qu'évoque Glück est ailleurs : le rôle du lecteur tel qu'elle le conçoit est à entendre dans la structure lyrique de l'adresse où la deuxième personne est convoquée, d'où la différence qu'elle suggère entre l'invitation des premiers vers de «The Love Song of J. Alfred Prufrock» de T.S. Eliot et celle du sonnet XVIII de Shakespeare :

[...] Eliot: "Let us go then, you and I,/When the evening is spread out against the sky/Like a patient etherized upon a table..." Eliot is not summoning the boyscout troop. He is asking something of the reader. As opposed, say, to Shakespeare's "Shall I compare thee to a summer's day": Shakespeare is not comparing me to a summer's day. I am being allowed to overhear dazzling virtuosity, but the poem does not require my presence. (Glück 2020)

Cette nuance dans la conception de l'apostrophe lyrique - objet de nombreux débats théoriques que je n'évoquerai pas ici - est cruciale pour Glück et aide à comprendre son propre ars poetica. La relation de complicité, voire de connivence que la poète cherche à créer avec chacun de ses lecteurs ouvre ses textes à « la possibilité d'une dialectique du désir " pour reprendre les fameux termes de Roland Barthes (Barthes 220), et se désintéresse, voire se méfie de ce qui pourrait survenir à l'extérieur de cet espace, espace magistralement mis en scène dans The Wild Iris (1992), recueil dont le dialogue triangulaire entre fleurs d'un jardin, jardinier et entité divine, structure une poétique de désir - le désir de survivre, de renaître, et simplement, de vivre (Glück, 1992 6) :

Do you know what I was, how I lived? You know

what despair is; then

winter should have meaning for you.

I did not expect to survive,

earth suppressing me. I didn't expect

to waken again, to feel

in damp earth my body

able to respond again, remembering

after so long how to open again

in the cold light

of earliest spring-

afraid, yes, but among you again

crying yes risk joy

in the raw wind of the new world.

("Snowdrops") $)^{2}$

6 L'un des pouvoirs d'attraction de l'œuvre de la poète américaine réside dans la lisibilité d'une langue vernaculaire dont les aspérités semblent pourtant toujours garder l'objet du dire en réserve ${ }^{3}$ à travers une écriture profondément mue par le désir d'esquiver les dichotomies, prenant, dans ses premiers recueils, la forme d'un cri de protestation contre la mort, puis celle d'un vouloir-vivre. Au fil de son œuvre, ce cri a su perdre de 
sa véhémence pour revêtir l'expression la plus naturelle : celle de la respiration à travers des poèmes de plus en plus longs, lieux de détour du sens où ce dernier suit parfois le tracé d'une parabole (genre que Glück affectionne particulièrement, à l'instar de la fable, notamment), mais jamais celui de l'assertion, toujours celui d'une interrogation qui happe le sens tout en le laissant en suspens. Au-delà d'une apparente simplicité de langue et de syntaxe, la poésie de Glück pose de nombreux problèmes dont l'écart entre ce que sa poétique donne plus ou moins ouvertement à lire et l'obscurité inhérente à ses signes, eux-mêmes lieux de ressac du temps et nœuds sémantiques.

7 Dans «Odysseus' Decision" par exemple (Meadowlands 1996), le tissu poétique fallacieusement lisse se révèle sous-tendu par une urgence lyrique, qui contribue à faire participer le lecteur à la construction du sens (Glück, 1996 42) :

The great man turns his back on the island.

Now he will not die in paradise

nor hear again

the lutes of paradise among the olive trees,

by the clear pools under the cypresses. Time

begins now, in which he hears again

that pulse which is the narrative

sea, at dawn when its pull is strongest.

What has brought us here

will lead us away; our ship

sways in the tinted harbor water.

Now the spell is ended.

Give him back his life,

sea that can only move forward.

Le poème met en scène l'instant liminaire précédant le départ, alors qu'Ulysse tourne le dos à Schérie avant de retourner à Ithaque. Le poème déploie dans une tranquillité presque déjà nostalgique une narrativité qui ne se veut pas prosaïque, mais lyrique, car installée dans le présent de l'écriture, et le pas encore de l'action. Comme le suggère la dernière strophe et le passage en italiques que l'on imagine être du discours direct - ou tout du moins l'expression d'une pensée -, le départ d'Ulysse est à la fois sa décision (comme l'indique le titre), sans pour autant véritablement lui appartenir. "Odysseus' Decision » explore l'oscillation d'une décision au bord de l'hésitation, au bord du nonchoix. Comme souvent dans sa poésie, la volonté se retrouve mise en suspens. Or, c'est la répétition anaphorique de ce «now» qui impulse toute l'énergie au poème, convoque le lecteur, exige de lui une participation active. C'est également à travers le rythme du poème, et au creux de ce blanc interstrophique que le plaisir du lecteur est ardemment convié, où le temps se trouve comme suspendu. À cet espace correspond étrangement le présent du poème, soupir avant le commencement («begins, " commence la deuxième strophe), avant l'écriture même. Ainsi seulement débuterait le voyage, au cœur du poème, après une respiration, après le vide.

8 Le monde poétique de Glück est un monde où la décision la plus radicale s'avère, dans l'écriture, détachée du sujet. Le sens y est souvent contenu, voire retenu, dans l'espace d'une oscillation, le temps d'une respiration. Ici, le blanc interstrophique déjà évoqué accumule le momentum, l'énergie du poème, avant de le relâcher au travers de la deuxième strophe : «that pulse, which is the narrative/sea ». Ce sont cette énergie et ce rythme vitaux que l'on trouve dans la succession des vers, avant le ressac de la dernière strophe (« Now the spell is ended./Give him back his life,/sea that can only 
move forward ») où l'injonction à la vie, au mouvement, vient inéluctablement, et sans alternative possible, à la persona comme au lecteur. Lumineuse, l'image de la mer narrative devient l'incarnation de celle qui raconte, déplie l'histoire, et propulse le voyageur, comme le lecteur, toujours en avant. Elle est également incarnation de l'origine, mère nourricière, qui rapatrie Ulysse à Ithaque - un Ulysse devenu Protée, prophète à toujours regarder derrière lui.

C'est à cette double injonction vitale que la poésie de Louise Glück semble avoir plus récemment obéi. Tout comme le héros de Homère, tournant le dos au plaisir, prêt à embarquer pour le voyage du retour, le moi lyrique glückien a graduellement su accepter le corps, l'humanité, ses faiblesses et ses défauts, comme on peut le lire dans Averno (Glück, 2006 52) :

I was trying to love matter.

I taped a sign over the mirror:

You cannot hate matter and love form.

[...]

List of things to love:

dirt, food, shells, human hair

("Archaic Fragment" $1-2 ; 10-11$ )

Le recours aux italiques permet de faire surgir la corporalité dans le langage et dans le texte. L'œuvre de la poète américaine résiste à l'objet - le « de quoi ça parle » - à toute thématisation et à toute tentative de définition.

Depuis le début de sa carrière, Louise Glück a su se réinventer constamment, explorer puis abandonner un style sans pour autant perdre la singularité de sa voix poétique. Ses deux derniers recueils, A Village Life (2009) et Faithful and Virtuous Night (2014) accueillent la vie comme la mort selon des modalités différentes, mais tous deux dans une sérénité grandissante. L'humour y est sans cesse présent, loin de la tourmente et de la vitalité désespérée des premiers recueils, comme ici dans Firstborn (1968), premier recueil que Glück a pu considérer comme un exercice de style (Glück, 19957 ; 10) :

My body stretching like a tear

Along the paper

("The Egg" part 2)

[...] And lived to see

That all that all flushed down

The refuse. Done?

It lives in me.

You live in me. Malignant.

Love, you ever want me, don't.

("Hesitate to Call" 4-9)

Depuis cette précision chirurgicale, cette austérité froide, et un état de stase initiale ses poèmes ont su vibrer, puis bouger, jusqu'à s'aventurer à donner l'illusion de raconter une histoire. Très vite (depuis 1985 avec The Triumph of Achilles), les poèmes se sont étirés au fil des pages et des recueils. Entre la verticalité ${ }^{5}$ de The House on Marshland (1975) et de Descending Figure (1980), et les longs sillons de Faithful and Virtuous Night (2014), les poèmes de Glück ont exploré avec ironie l'expérience du deuil dans Ararat (1990), fait dialoguer les fleurs d'un jardin avec leurs créateurs dans The Wild Iris (1992), réécrit l'odyssée dans Meadowlands (1996), et évoqué le sujet de la disparition au fil des saisons au travers des longs poèmes d'Averno (2006) au lyrisme mahlérien.

11 Plus récemment, Glück a pu surprendre ses lecteurs, comme par exemple dans A Village Life (2009) dont l'atmosphère de fin du monde est perçue par une myriade de 
personnages dans une tonalité étonnamment sereine. De la même façon, Faithful and Virtuous Night introduit pour la première fois des poèmes en prose et explore un rythme plus long, plus narratif sans jamais l'être tout à fait ${ }^{6}$. La poétique de Glück semble être mue par une énergie née de l'esquive de paradigmes, de pôles opposés (Glück, 2014 62) :

I closed my eyes.

I was torn between a structure of oppositions

and a narrative structure-

("The Story of a Day" 41-43)

La persona glückienne se construit à travers l'expérience de son environnement, et sa relation à la figure de l'autre (qu'il s'agisse de la nature, de l'être aimé, etc.). L'instance lyrique figure le nœud du chiasme entre le dehors et le dedans, entre introspection et ouverture au monde. Souvent, les personae ressentent le besoin de se projeter et de s'identifier dans cet ailleurs qu'est l'autre avant de pouvoir seulement envisager d'exister. C'est dans cette relation essentielle avec l'altérité que le désir s'installe et que sa poésie peut se développer. Il ne s'agit pas nécessairement du désir amoureux, mais du désir d'exister, de trouver la paix intérieure, qui passe fréquemment chez elle par un désir d'écriture. Reviennent souvent dans ses recueils des poèmes insulaires, lieux de méditation, où le monde semble mis en suspens, et où l'instance lyrique semble atteindre l'ataraxie un bref instant, comme ici dans «Eros» (The Seven Ages, Glück, $200158)$ :

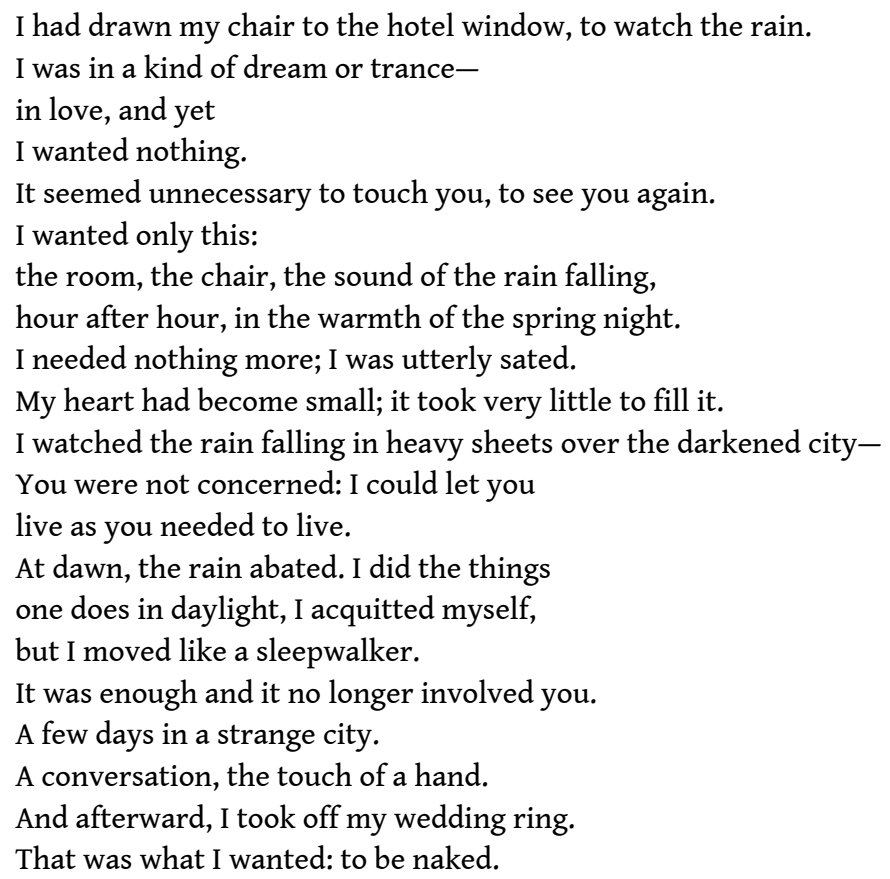

12 Le sentiment de calme et de paix qui habite le poème est saisissant et envahissant. Si le désir anime chaque vers, il s'avère paradoxalement explicité par deux verbes seulement : «want » (4) et «need» (9). Cependant, ces derniers sont immédiatement neutralisés, restreints syntaxiquement car adjoints d'un adverbe, ou simplement rayés par une négation dans les vers qui suivent. L'objet du désir paraît avoir été gommé de la page sans pour autant en avoir été abstrait. À aucun instant la voix ne permet au désir de le submerger de façon sentimentale ou par un quelconque pathos. Et pourtant, ce désir hante le poème à la manière d'un fantôme sur des ruines, c'est-à-dire en matérialisant son absence. Souvent de nature complexe, comme ici dans «Eros», il 
semble détaché de l'objet, intransitif, à l'image de son écriture, qui s'avère être souvent à la fois l'objet et le telos de ses poèmes (Glück, 2006 62) :

my breath was white, a description of silence.

Time passed, and some of it became this.

And some of it simply evaporated;

you could see it float above the white trees

forming particles of ice.

All your life, you wait for the propitious time.

Then the propitious time

reveals itself as action taken.

(“Averno" 47-54)

L'écriture procède paradoxalement ici d'un geste en suspens, à la fois immergé dans le temps et à l'écart de lui : « it was a time/of waiting, of suspended action.//I lived in the present, which was/that part of the future you could see. The past floated above my head,/like the sun and moon, visible but never reachable " (34-39). Cette temporalité relève d'un kairos, instant crucial où se joue l'écriture, et par lequel il n'est déjà plus temps - l'écriture étant déjà survenue : «then the propitious time/reveals itself as action taken ».

A Village Life et Faithful and Virtuous Night continuent de travailler l'oscillation entre les dichotomies, mais en travaillant une temporalité plus longue, en aménageant cette langue davantage narrative, qui se veut également temps de l'écriture.

Comme le vaisseau de l'Argo, l'œuvre de la poète se renouvelle sans cesse. Et pourtant, la voix que l'on y entend demeure singulière, reconnaissable entre toutes. Le spirituel et le mythologique y côtoient le monde matériel, la quotidienneté de l'existence, comme ici, dans The Wild Iris (Glück, 1992 36) :

Once I believed in you; I planted a fig tree.

Here, in Vermont, country

of no summer. It was a test: if the tree lived,

it would mean you existed.

By this logic, you do not exist. Or you exist

exclusively in warmer climates,

in fervent Sicily, and Mexico and California,

where are grown the unimaginable

apricot and fragile peach. Perhaps

they see your face in Sicily; here, we barely see

the hem of your garment. I have to discipline myself

to share with John and Noah the tomato crop.

("Vespers" 1-12)

Chez Glück, la croyance semble jusqu'au bout enchainée au doute ; elle lui résiste et lui survit, comme s'il en était finalement la condition.

De plus, quel que soit le recueil, quel que soit le désespoir qui l'habite, l'humour et l'ironie ne sont jamais très loin. Glück nous entraîne dans un jeu d'équilibriste, et c'est cette oscillation entre les modes et les tonalités qui contribue à la jubilation du lecteur, pris par surprise, comme dans ces quelques vers d'Ararat ou de Meadowlands :

My mother wants to know

why, if I hate

family so much,

I went ahead and

had one. I don't

answer my mother.

("Brown Circle" Ararat 42, 1-6) 


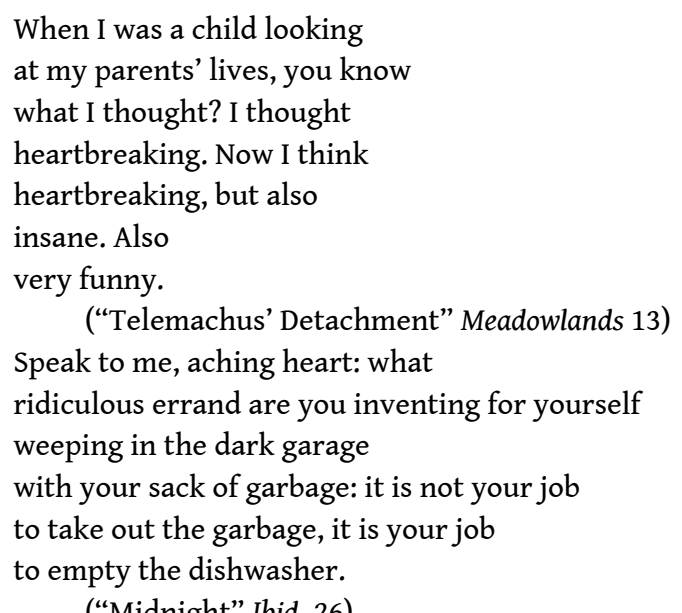

("Midnight" Ibid. 26)

C'est précisément parce que « de quoi ça parle » importe peu, que sa poésie nous parle tant. Chaque recueil de Louise Glück mène à un endroit imprévu, étonne de maintes façons différentes: la diversité dans la tonalité, les changements de rythme, de respiration, l'équilibre ou le déséquilibre entre image et abstraction. Autant de traits caractéristiques qui convoquent la présence et le corps même du lecteur, suscitent son enthousiasme et créent à chaque fois en lui le désir du recueil à venir ${ }^{7}$.

\section{BIBLIOGRAPHIE}

BARTHES, Roland. « Le Plaisir du Texte ». 1973. Cuvres Complètes IV. Paris : Seuil, 2002, p. 217-266.

GLÜCK, Louise. Ararat. New York : Ecco Press, 1990.

GLÜCK, Louise. The Wild Iris. New York : Ecco Press, 1992.

GLÜCK, Louise. Firstborn in The First Four Books of Poems. New York : Ecco Press, 1995.

GLÜCK, Louise. Meadowlands. New York : HarperCollins, 1996.

GLÜCK, Louise. Averno. Manchester : Carcanet Press, 2006.

GLÜCK, Louise. A Village Life. New York : Farrar, 2009.

GLÜCK, Louise. Faithful and Virtuous Night. Manchester : Carcanet Press, 2014.

GLÜCK, Louise. "Nobel Lecture by Louise Glück, Nobel Laureate in Literature 2020.” The Nobel Foundation 2020. www.nobelprize.org/prizes/literature/2020/gluck/lecture/. Consulté le 15 juin 2021.

OLIVIER, Marie. « Le chant en réserve, lecture de deux poèmes de Louise Glück », L'Atelier 7:2 (2015), p. 23-42. ojs.u-paris10.fr/index.php/latelier/article/view/433. Consulté le 15 juin 2021.

OLIVIER, Marie. « The Mutable Poetics of Louise Glück, A Reading of "Summer Garden” by Louise Glück ». Angles, Unstable states, mutable conditions, ${ }^{\circ} 4$ |2017, https://journals.openedition.org/ angles/1516. Consulté le 15 juin 2021. 


\section{Bibliographie sélective de Louise Glück}

\section{Recueils de poésie}

GLÜCK, Louise. Ararat. New York: Ecco Press, 1990.

GLÜCK, Louise. The Wild Iris. New York: Ecco Press, 1992.

GLÜCK, Louise. The First Four Books of Poems. New York: Ecco Press, 1995.

GLÜCK, Louise. Meadowlands. New York: HarperCollins, 1996.

GLÜCK, Louise. Vita Nova. New York: Ecco Press, 1999.

GLÜCK, Louise. The Seven Ages. New York: Ecco Press, 2001.

GLÜCK, Louise. Averno. Manchester: Carcanet Press, 2006.

GLÜCK, Louise. A Village Life. New York: Farrar, 2009.

GLÜCK, Louise. Poems 1962-2012. New York: Farrar Straus and Giroux, 2012.

GLÜCK, Louise. Faithful and Virtuous Night. Manchester: Carcanet Press, 2014.

\section{Recueil d'essais}

GLÜCK, Louise. Proofs and Theories. Hopewell: Ecco Press, 1994.

GLÜCK, Louise. American Originality: Essays on Poetry. New York: Farrar Straus and Giroux, 2017.

\section{NOTES}

1. Contrairement aux usages, et en raison des conditions sanitaires, Louise Glück ne s'est pas rendue à Stockholm pour prononcer son discours.

2. Les poèmes «Snowdrops » (The Wild Iris, 1992), "Odysseus' Decision » (Meadowlands, 1996) et «Eros» (The Seven Ages, 2001) sont ici reproduits dans leur intégralité avec l'aimable autorisation de Carcanet Press, Manchester, UK.

3. À ce sujet, lire «Le chant en réserve, lecture de deux poèmes de Louise Glück » (Olivier 2015).

4. On peut le constater jusqu'à The Triumph of Achilles (1985).

5. Cette verticalité renvoie non seulement à la façon dont le poème se déploie visuellement sur la page, mais aussi à son mouvement.

6. Sur la narration dans Faithful and Virtuous Night, lire « The Mutable Poetics of Louise Glück, A Reading of 'Summer Garden' by Louise Glück » (Olivier 2017).

7. Le prochain recueil de Louise Glück, Winter Recipes from the Collective, sort à l'automne prochain chez Farrar, Straus and Giroux.

\section{AUTEUR}

\section{MARIE OLIVIER}

Université Paris-Est Créteil. IMAGER EA 3958 